\title{
El liderazgo directivo y el desempeño docente en el nivel secundaria en las instituciones educativas públicas del distrito de Pachacámac de la UGEL 01, 2018.
}

\section{The management leadership and teaching performance at the secondary level in the public educational institutions of the Pachacámac district of the UGEL 01, 2018.}

\author{
Melissa Mónica Segil Rodríguez
}

\section{RESUMEN}

Objetivo: Determinar la relación entre el liderazgo directivo y el desempeño docente en el nivel secundaria en las instituciones educativas públicas del distrito de Pachacámac de la UGEL 01, 2018. Método: La investigación es de tipo básica, descriptiva y correlacional con diseño no experimental; la muestra estuvo conformada por 24 directivos, 72 docentes y 94 estudiantes del 4 to y 5 to grado del nivel secundaria de las 15 instituciones educativas públicas del distrito de Pachacámac, se aplicó una guía de entrevista para los directivos, un cuestionario para los docentes y otro cuestionario para los estudiantes. Resultados: Se comprobó la relación entre las variables y la comprobación de las hipótesis planteadas demostrando que existe relación entre el liderazgo directivo y el desempeño docente en el nivel secundaria en las instituciones educativas públicas del distrito de Pachacámac de la UGEL 01, 2018. Conclusión: El liderazgo directivo presenta una relación significativa con el desempeño docente en el nivel secundaria en las instituciones educativas públicas del distrito de Pachacámac de la UGEL 01, 2018.

Palabras claves: liderazgo directivo, desempeño docente

\begin{abstract}
Objective: To determine the relationship between managerial leadership and teacher performance at the secondary level in the public educational institutions of the Pachacámac district of UGEL 01, 2018. Method: The research is basic, descriptive and correlational with non-experimental design; the sample consisted of 24 executives, 72 teachers and 94 students of the 4th and 5th grade of the secondary level of the 15 public educational institutions of the Pachacámac district, an interview guide was applied for the directors, a questionnaire for the teachers and another questionnaire for the students. Results: The relationship between the variables and the verification of the hypotheses raised was demonstrated by demonstrating that there is a relationship between the leadership and the teaching performance at the secondary level in the public educational institutions of the Pachacámac district of the UGEL 01, 2018. Conclusion: The leadership leadership presents a significant relationship with the teaching performance at the secondary level in the public educational instituti chacámac district of the UGEL 01, 2018
\end{abstract}

Keywords: management lidership, performance teacher

\section{INTRODUCCION}

En la actualidad, la educación a nivel mundial viene sufriendo cambios y la necesidad de responder con éxito a las demandas de la sociedad ha hecho que el liderazgo directivo se constituya en un tema estratégico dentro de las políticas educativas, considerado un factor escolar vinculado a la mejora del desempeño docente y por ende la mejora de los logros de aprendizaje. Para ello es necesario contar con directivos que sean lideres pedagógicos que promuevan el crecimiento personal y profesional del equipo docente a través de la efectividad en las prácticas de liderazgo directivo y así puedan favorecer los aprendizajes de calidad de los estudiantes.

Castañeda (2005), señala que el "liderazgo es el conjunto de cualidades y hábitos positivos que motivan y permiten a un individuo conducir a un grupo de personas al logro de fines superiores por caminos acotados por principios y valores de alto contenido moral" (p.21). Así también Hunt (2009), nos habla tanto de un liderazgo efectivo como de una docencia efectiva que implica un conjunto de habilidades y competencias que se adquieren, para ello es necesario proveer a los directivos de herramientas en los programas de actualización y capacitación que contribuyan a desarrollar tales competencias realizando el acompañamiento, monitoreo y supervisión efectiva a los docentes. Para Bolívar (2010), la habilidad para mejorar una institución educativa obedece al ejercicio del liderazgo de los equipos directivos contribuyendo a erigir la capacidad interna de mejora dinamizando, alentando y ayudando a que la organización aprenda a desarrollarse.

Leithwood (2009), menciona cuatro dimensiones de prácticas de un liderazgo escolar efectivo, las cuales son: establecer direcciones, desarrollar personas, rediseñar la organización, y gestionar la instrucción. Anderson (2010), menciona que: establecer direcciones influye relevantemente sobre el compromiso y disposición de los docentes a mejorar su desempeño, el desarrollo de personas contribuye a la mejora de competencias profesionales de los docentes y rediseñar la organización y gestionar la instrucción están dirigidas a enriquecer las condiciones de trabajo de los docentes.

Castro (2015), sostiene: "La competencia docente se entiende como la movilización de recursos intelectuales profesionales para utilizar determinados objetos de aprendizaje mediante metodologías didácticas pertinentes con profesionalismo ético en un contexto definido" (p.265). Así para (Cuenca, Montero,

Recibido:4/03/2020 - Aprobado:19/08/2020 
Ames, \& Rojas, 2011), un buen desempeño docente está relacionado a una buena práctica pedagógica y metodológica permitiendo que los estudiantes disfruten del proceso enseñanza aprendizaje.

MINEDU (2014), refiere cuatro dominios en el Marco del Buen Desempeño Docente: preparación para el aprendizaje de los estudiantes, enseñanza para el aprendizaje de los estudiantes, participación en la gestión de la escuela articulada a la comunidad y desarrollo de la profesionalidad y la identidad docente.

El objetivo de esta investigación fue determinar la relación entre el liderazgo directivo y el desempeño docente en el nivel secundaria en las instituciones educativas públicas del distrito de Pachacámac de la UGEL 01, 2018.

\section{MATERIALES Y MÉTODOS}

La investigación es de tipo básica, descriptiva y correlacional con diseño no experimental. La muestra estuvo conformada por 190 personas, entre ellas 24 directivos, 72 docentes y 94 estudiantes del 4 to y 5 to grado del nivel secundaria de las 15 instituciones educativas públicas del distrito de Pachacámac; para la selección de la muestra, en cuanto a los directivos se realizó un censo y el muestreo estratificado proporcional para los docentes y estudiantes.

Se aplicaron tres instrumentos, una guía de entrevista para los directivos y un cuestionario para los docentes para medir las variables liderazgo directivo con 34 ítems y desempeño docente con 39 ítems. Un tercer instrumento con 20 ítems aplicado a los estudiantes para medir la variable desempeño docente. Los instrumentos han sido validados mediante juicio de expertos y presentan un adecuado nivel de confiabilidad: 0,$951 ; 0,965$ y 0,870 respectivamente. Se usó el programa IBM SPSS Statistics 25.0 en la sistematización de datos, el estudio cuantitativo de datos y la interpretación de resultados.

\section{RESULTADOS}

1.Existe relación entre el liderazgo directivo y el desempeño docente en el nivel secundaria en las instituciones educativas públicas del distrito de Pachacámac de la UGEL 01, 2018.

Tabla 1: Relación entre el liderazgo directivo y el desempeño docente

\begin{tabular}{llrr}
\hline & & $\begin{array}{l}\text { LIDERAZGO } \\
\text { DIRECTIVO }\end{array}$ & \multicolumn{2}{c}{$\begin{array}{c}\text { DESEMPEÑO } \\
\text { DOCENTE }\end{array}$} \\
& & 1 &, $826^{* *}$ \\
LIDERAZGO & Correlación de Pearson & 960 \\
DIRECTIVO & Sig.(bilateral) & 96 \\
& $\mathrm{~N}$ & & \\
DESEMPEÑO & Correlación de Pearson &, $826^{* *}$ & 1 \\
DOCENTE & Sig.(bilateral) &, 000 & 96 \\
& $\mathrm{~N}$ & 96 & \\
\hline
\end{tabular}

Nota: Base de datos IBM SPSS Statistics 25.0

Conclusión: Se demostró una relación significativa entre el liderazgo directivo y el desempeño docente en el nivel secundaria en las instituciones educativas públicas del distrito de Pachacámac de la UGEL 01, 2018, considerado como correlación positiva fuerte, esto significa que a mayor o menor liderazgo directivo, mayor o menor será el desempeño docente.

1.Existe relación entre el liderazgo directivo y la preparación para el aprendizaje de los estudiantes en el nivel secundaria en las instituciones educativas públicas del distrito de Pachacámac de la UGEL 01, 2018.

Tabla 2: Relación entre el liderazgo directivo y la preparación para el aprendizaje de los estudiantes

\begin{tabular}{llrr}
\hline & & $\begin{array}{l}\text { LIDERAZGO } \\
\text { DIRECTIVO }\end{array}$ & $\begin{array}{l}\text { DESEMPEÑO } \\
\text { DOCENTE }\end{array}$ \\
\hline LIDERAZGO & Correlación de & 1 & \\
DIRECTIVO & $\begin{array}{l}\text { Pearson } \\
\text { Sig.(bilateral) }\end{array}$ & &, $732^{* *}$ \\
& $\mathrm{~N}$ & &, 000 \\
& & 96 & 96 \\
PREPARACION & Correlación de &, $732^{* *}$ & 1 \\
PARA EL & Pearson & & \\
APRENDIZAJE & Sig.(bilateral) &, 000 & \\
DEL OS & $\mathrm{N}$ & 96 & 96 \\
ESTTUDIANTES & & & \\
\hline
\end{tabular}

Nota: Base de datos IBM SPSS Statistics 25.0

Conclusión: Se estableció una relación significativa entre el liderazgo directivo y la preparación para el aprendizaje de los estudiantes en el nivel secundaria en las instituciones educativas públicas del distrito de Pachacámac de la UGEL 01 , 2018, considerado como correlación positiva media, esto significa que a mayor o menor liderazgo directivo, mayor o menor será la preparación para el aprendizaje de los estudiantes.

1.Existe relación entre el liderazgo directivo y la enseñanza para el aprendizaje de los estudiantes en el nivel secundaria en las instituciones educativas públicas del distrito de Pachacámac de la UGEL 01, 2018.

Tabla 3: Relación entre el liderazgo directivo y la enseñanza para el aprendizaje de los estudiantes

$\begin{array}{ll}\text { LIDERAZGO } & \text { ENZEÑANZA } \\ \text { DIRECTIVO } & \text { PARA EL } \\ & \text { APRENDIZAJE } \\ & \text { DE LOS } \\ & \text { ESTUDIANTES }\end{array}$

\begin{tabular}{llrr}
\hline LIDERAZGO & Correlación de & 1 &, $704^{* *}$ \\
DIRECTIVO & $\begin{array}{l}\text { Pearson } \\
\text { Sig.(bilateral) }\end{array}$ & &, 000 \\
& $\mathrm{~N}$ & 96 & 96 \\
& & & 1 \\
ENZEÑANZA & Correlación de &, $704^{* *}$ & \\
PARA EL & Pearson & & \\
APRENDIZAJE DE & Sig.(bilateral) &, 000 & 96 \\
LOS & $\mathrm{N}$ & 96 & \\
ESTUDIANTES & & & \\
\hline
\end{tabular}

Nota: Base de datos IBM SPSS Statistics 25.0 
Conclusión: Se demostró una relación significativa entre el liderazgo directivo la enseñanza para el aprendizaje de los estudiantes en el nivel secundaria en las instituciones educativas públicas del distrito de Pachacámac de la UGEL 01 , 2018, considerado como correlación positiva media, esto significa que a mayor o menor liderazgo directivo, mayor o menor será la enseñanza para el aprendizaje de los estudiantes.

1.Existe relación entre el liderazgo directivo y la participación en la gestión de la escuela articulada a la comunidad en el nivel secundaria en las instituciones educativas públicas del distrito de Pachacámac de la UGEL 01, 2018.

Tabla 4: Relación entre el liderazgo directivo y la participación en la gestión de la escuela articulada a la comunidad

\begin{tabular}{|c|c|c|c|}
\hline & & $\begin{array}{l}\text { LIDERAZGO } \\
\text { DIRECTIVO }\end{array}$ & $\begin{array}{l}\text { PARTICIPACIÓN } \\
\text { EN LA } \\
\text { GESTIÓN DE } \\
\text { LA ESCUELA } \\
\text { ARTICULADA }\end{array}$ \\
\hline LIDERAZGO & Correlación de & 1 &, $704^{* *}$ \\
\hline \multirow[t]{3}{*}{ DIRECTIVO } & Pearson & & \\
\hline & Sig.(bilateral) & &, 000 \\
\hline & $\mathrm{N}$ & 96 & 96 \\
\hline PARTICIPACIÓN & Correlación de &, $704^{\star *}$ & 1 \\
\hline EN LA GESTIÓN & Pearson & & \\
\hline DE LA ESCUELA & Sig.(bilateral) &, 000 & \\
\hline ARTICULADAA LA & $\mathrm{N}$ & 96 & 96 \\
\hline COMUNIDAD & & & \\
\hline
\end{tabular}

Nota: Base de datos IBM SPSS Statistics 25.0

Conclusión: Se demostró que existe una relación significativa entre el liderazgo directivo y la participación en la gestión de la escuela articulada a la comunidad en el nivel secundaria en las instituciones educativas públicas del distrito de Pachacámac de la UGEL 01, 2018, considerado como correlación positiva media, esto significa que a mayor o menor liderazgo directivo, mayor o menor será la participación en la gestión de la escuela articulada a la comunidad.

1.Existe relación entre el liderazgo directivo y el desarrollo de la profesionalidad y la identidad docente en el nivel secundaria en las instituciones educativas públicas del distrito de Pachacámac de la UGEL 01, 2018.
Tabla 5: Relación entre el liderazgo directivo y el desarrollo de la profesionalidad y la identidad docente

\begin{tabular}{|c|c|c|c|}
\hline & & $\begin{array}{l}\text { LIDERAZGO } \\
\text { DIRECTIVO }\end{array}$ & $\begin{array}{l}\text { DESARROLLO } \\
\text { DE LA } \\
\text { PROFESIONALI } \\
\text { DAD Y LA } \\
\text { IDENTIDAD } \\
\text { DOCENTE }\end{array}$ \\
\hline LIDERAZGO & Correlación de & 1 &, $728^{* *}$ \\
\hline \multirow{3}{*}{ DIRECTIVO } & Pearson & & \\
\hline & Sig.(bilateral) & & ,000 \\
\hline & $\mathrm{N}$ & 96 & 96 \\
\hline DESARROLLO DE & Correlación de &, $728^{\star *}$ & 1 \\
\hline LA & Pearson & & \\
\hline PROFESIONALIDAD & Sig.(bilateral) & ,000 & \\
\hline Y LA IDENTIDAD & $\mathrm{N}$ & 96 & 96 \\
\hline DOCENTE & & & \\
\hline
\end{tabular}

Nota: Base de datos IBM SPSS Statistics 25.0

Conclusión: Se estableció una relación significativa entre el liderazgo directivo y el desarrollo de la profesionalidad y la identidad docente en el nivel secundaria en las instituciones educativas públicas del distrito de Pachacámac de la UGEL 01, 2018, considerado como correlación positiva media, esto significa que a mayor o menor liderazgo directivo, mayor o menor será el desarrollo de la profesionalidad y la identidad docente.

\section{DISCUSIÓN}

De acuerdo a los resultados de la investigación se puede establecer similitudes con la teoría y los antecedentes de investigaciones similares. Se encuentra una relación significativa entre el liderazgo directivo y el desempeño docente en el nivel secundaria de las instituciones educativas públicas. Los resultados reafirman lo encontrado por:

Leithwood, Seashore Louis, Anderson \& Wahlstrom (2004), señalan que "el liderazgo es el segundo factor intra-escuela, después del trabajo docente en sala de clases, que más contribuye al logro de aprendizajes de los alumnos" (p.3).

Zárate (2011), señala la existencia de un alto grado de correlación lineal entre el liderazgo directivo y el desempeño docente con sus respectivas dimensiones. El director lidera en su labor en las dimensiones: gestión pedagógica, institucional y administrativo como consecuencia el desempeño docente en sus dimensiones: profesional, personal y social resulta óptimo, de la misma forma alcanza ocurrir a la inversa.

Raxuleu (2014), indica que existe una vinculación lineal directa entre las variables de liderazgo del director y desempeño pedagógico docente. Una amplia mayoría de respuestas obtenidas apuntan a que el desenvolvimiento de los directores mediante cada acción afecta o influye en el accionar pedagógico de los docentes en las aulas. La vinculación entre las dos variables es alta.

Rojas (2017), afirma que existe una correlación moderada entre las variables liderazgo del director respecto al desempeño docente repercutiendo en la imagen de la I.E Javier Heraud de San Juan de Miraflores donde se realizó la 
investigación.

\section{AGRADECIMIENTOS}

A todos los directivos, docentes y estudiantes de las instituciones educativas públicas del distrito de Pachacámac por su valiosa colaboración en la realización de esta investigación.

\section{REFERENCIAS BIBLIOGRÁFICAS}

Castañeda Martínez, L. (2005). Un Plan de Formación en Liderazgo parajóvenes. (E. PODER, Ed.) Panorama.

Cuenca, R., Montero, C., Ames, P., \& Rojas, V. (2011). Hacia una propuesta de criterios de buen desempeño. Estudios que aportan a la reflexión, al diálogo y a la construcción concertada de una política educativa. (C. N. Educación, Ed.) Lima, Perú.

Leithwood, K. (2009). ¿Cómo liderar nuestras escuelas? Aportes desdela investigación. Santiago de Chile: Área de Educación Fundación Chile.

Leithwood, K., Seashore Louis, K., Anderson, S., \& Wahlstrom, K.(2004). How leadership influences student learning: A review of research for the learning fro $\mathrm{m}$ leadership project. New York, NY: The Wallace Foundation.

MINEDU. (2014). Marco del Buen Desempeño Docente. Lima, Perú.

Rojas Landa, V. C. (2017). EL liderazgo del director y el desempeñodocente en la Institución Educativa Javier Heraud del distrito de San Juan de Miraflores 2014. Tesis para optar el grado académico de Magíster en Educación con Mención en Gestión de la Educación, Universidad Nacional Mayor de San Marcos. Lima, Perú.

Zarate Ramírez, D. (2011). Liderazgo directivo y el desempeño docenteen instituciones educativas de primaria del distrito de Independencia, Lima. Te s is para optar el grado académico de Magíster en Educación con Mención en Administración de la Educación Universitaria, Universidad Nacional Mayor de San Marcos. Lima, Perú.

Anderson, S. (2010). Liderazgo directivo: Claves para una mejor escuela. Psicoperspectivas. Individuo y Sociedad, 9(2). Recuperado el 13 de febrero de 2019, de http://www.psicoperspectivas.cl/index.php/psicopers pectivas/article/view/127/141.

Bolívar, A. (2010). El liderazgo educativo y su papel en la mejora: una revisión actual de sus posibilidades $y$ limitaciones. Psicoperspectivas. Individuo y Sociedad, 9(2). Recuperado el 13 de febrero de 2019, d

https://www.psicoperspectivas.cl/index.php/psicoper spectivas/article/view/112/140.
Castro Valles, A. (2015). Competencias Administrativas y Académicas en el Profesorado de Educación Media Superior. Revista Mexicana de Investigación Educativa, 20(64), 263-294. Recuperado el 15 de setiembre de 2019 , d e http://www.scielo.org.mx/pdf/rmie/v20n64/v20n64a1 3.pdf.

Hunt, B. (2009). Efectividad del desempeño docente. Una reseñade la literatura internacional y su relevancia para mejorar la educación en América Latina (1ra ed.). Santiago, Chile: San Marino. Recuperado el 5 de abril de 2018, de http://repositorio.minedu.gob.pe/bitstream/handle/12 $\begin{array}{llllllllllllllllll}3 & 4 & 5 & 6 & 7 & 8 & 9 & / & 3 & 0 & 9 & 3 & / & \mathrm{H} & \mathrm{u} & \mathrm{n} & \mathrm{t}\end{array}$ Efectividad\%20del\%20desempe\%c3\%b10\%20doce

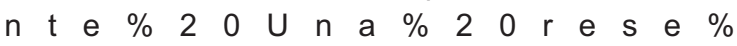
c3\%b1a\%20de\%20la\%20literatura\%20internacional

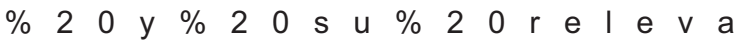
ncia $\% 20$ para $\% 20$ mejorar $\% 20 l a \% 20$ educaci $\%$ c3\%b $3 \mathrm{n} \% 20 \mathrm{en} \% 20 \mathrm{Am} \% \mathrm{c3} \%$ a9rica.

Raxuleu Ambrocio, M. (2014). Liderazgo del director y desempeñopedagógico docente. Sololá, Guatemala. Recuperado el 15 de noviembre de 2019, d http://recursosbiblio.url.edu.gt/tesiseortiz/2014/05/09 /Raxuleu-Manuel.pdf. 